\title{
Acute abdomen due to gastric volvulus: diagnostic value of a single plain radiograph
}

\author{
F.Andiran ${ }^{1}$, F. C. Tanyel ${ }^{1}$, F. Balkanci ${ }^{2}$, A.Hiçsönmez ${ }^{1}$ \\ ${ }^{1}$ Department of Pediatric Surgery, Hacettepe Children's Hospital, TR-06100, Ankara, Turkey \\ ${ }^{2}$ Department of Radiology, Hacettepe Children's Hospital, TR-06100, Ankara, Turkey
}

Received: 19 January 1995/Accepted: 20 April 1995

\begin{abstract}
A $2 \frac{1}{2}$-year-old child was admitted to hospital with acute abdominal pain and vomiting. A single large air-fluid level without additional bowel gas was seen on plain abdominal radiography. At laparotomy organoaxial volvulus of the stomach was found and partial gastric resection performed. A "single bubble" appearance may indicate gastric volvulus.
\end{abstract}

Because the stomach is relatively well fixed in the abdomen, gastric volvulus is a rare cause of acute abdominal pain and vomiting. Organoaxial, mesenteroaxial or mixed types of gastric volvulus may occur in cases of deficient fixation, diaphragmatic herniation and eventration and gastric distention [1-3]. Although it is known that torsion beyond $180^{\circ}$ may cause strangulation, gastric necrosis causing acute abdominal pain and vomiting is seldom reported in the literature $[3,4]$. A child who suffered gastric volvulus is presented to discuss the diagnostic value of plain radiographs.

\section{Case reports}

A $2 \frac{1}{2}$-year-old girl was admitted to the Department of Pediatric Surgery of Hacettepe Children's Hospital with abdominal pain, retching and vomiting which had begun $12 \mathrm{~h}$ prior to admission. The patient's past history did not reveal any previous similar episode. The physical examination revealed abdominal distention, tenderness and guarding. Apart from an elevated white blood cell count, the results of routine laboratory investigations including haemoglobin urinalysis, serum electrolyte levels and blood gases were within normal limits. Erect plain abdominal radiographs revealed

Correspondence to: F. C. Tanyel a single large air-fluid level without any diaphragmatic abnormality (Fig. 1). No gas was seen in other parts of the abdomen. Following initial fluid resuscitation, the patient underwent emergency laparotomy with the provisional diagnosis of gastric volvulus. Surgical exploration revealed an organoaxial volvulus of more than $180^{\circ}$ without any associated diaphragmatic pathology. Gastrosplenic and gastrocolic ligaments were present but lax. The volvulated part of the stomach, especially the fundus, was gangrenous and was resected. Postoperative recovery was uneventful.

\section{Discussion}

In patients admitted with abdominal pain, the main role of the radiologist is to recognise the patients requiring emergency operation. Since plain abdominal radiography may indicate the cause of acute abdominal pain, it is often the first examination of the routine investigation [5]. "Previous reports on gastric volvulus state that detection of a single air-fluid level on a plain abdominal radiograph suggests organoaxial gastric volvulus, however, barium swallow is usually suggested for confirmation [1-3]. The patient we present was admitted with the signs of acute abdomen. Since other conditions, such as pylqric obstruction which may have the same appearance on plain film, do not cause this condition, acute gastric volvulus was suspected. Therefore, confirmation by barium swallow was not obtained. The surgical exploration revealed gastric volvulus complicated with gangrene.

We suggest that a plain abdominal radiograph showing a large single airfluid level without any additional air bubble in patients admitted with acute abdomen should be considered suggestive of acute organoaxial gastric volvulus.

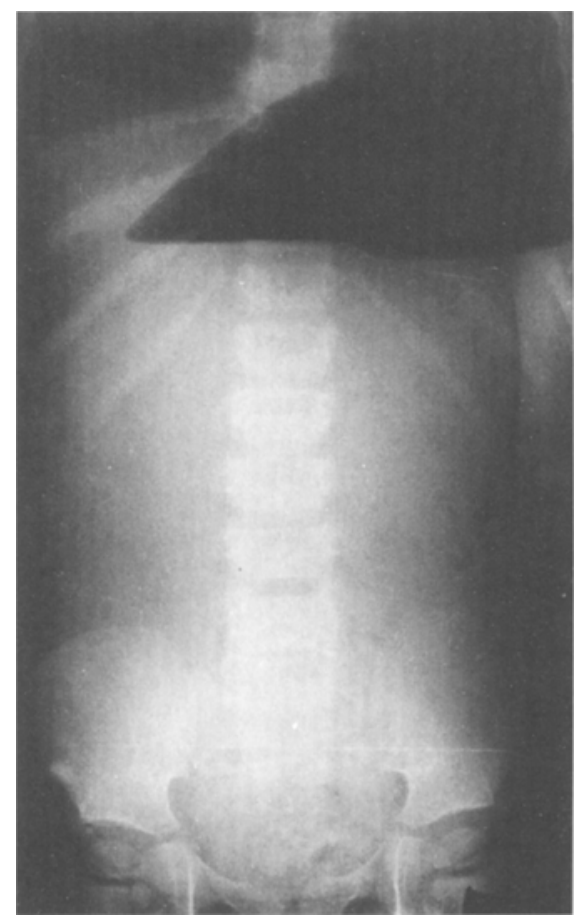

Fig. 1. A single large air-fluid level without any diaphragmatic pathology

\section{References}

1. Amin El-Gohary M, Etiaby A (1994) Gastric volvulus in infants and children. Pediatr Surg Int 9: 486-488

2. Cameron AEP, Howard ER (1987) Gastric volvulus in childhood. J Pediatr Surg 22: 944-947

3. Ziprkowski MN, Teele RT (1979) Gastric volvulus in childhood. AJR 132: 921-925

4. Carter R, Brewer LA, Hinshaw DB (1980) Acute gastric volvulus. Am J Surg 140: 99-106

5. Idowu J, Aitken DR, Georgeson KE (1980) Gastric volvulus in the newborn. Arch Surg 115: 1046-1049 